\title{
Supplementation with Korean Red Ginseng Improves Current Perception Threshold in Korean Type 2 Diabetes Patients: A Randomized, Double-Blind, Placebo-Controlled Trial
}

\author{
Kahui Park $\mathbb{D}^{1}{ }^{1}$ YuSik Kim, ${ }^{1,2}$ Junghye Kim, ${ }^{1}$ Shinae Kang $\mathbb{D}^{1,2}$ Jong Suk Park $\mathbb{D}^{1,2}$ \\ Chul Woo Ahn, ${ }^{1,2}$ and Ji Sun Nam $\mathbb{1}^{1,2}$ \\ ${ }^{1}$ Division of Endocrinology, Department of Internal Medicine, Gangnam Severance Hospital, \\ Yonsei University College of Medicine, Republic of Korea \\ ${ }^{2}$ Severance Institute for Vascular and Metabolic Research, Yonsei University College of Medicine, Republic of Korea \\ Correspondence should be addressed to Ji Sun Nam; jisunn@yuhs.ac
}

Received 18 September 2019; Accepted 7 January 2020; Published 18 January 2020

Guest Editor: Ruozhi Zhao

Copyright (c) 2020 Kahui Park et al. This is an open access article distributed under the Creative Commons Attribution License, which permits unrestricted use, distribution, and reproduction in any medium, provided the original work is properly cited.

\begin{abstract}
Background. Many Type 2 diabetes (T2DM) patients in Korea take Korean Red Ginseng (KRG) for various reasons. In this study, we investigated the effects of KRG administration on diabetic peripheral neuropathy in T2DM patients. Methods. This study was a randomized, double-blind, placebo-controlled trial. Participants were randomly allocated to either the placebo or KRG group and took corresponding tablets for 24 weeks. The primary outcomes were changes in current perception threshold (CPT) at week 24 . Secondary outcomes were altered fasting plasma glucose, HbAlc, and various metabolic and inflammatory markers at week 24. Results. Sixty-one patients completed the study. The CPT of the lower extremities at various frequencies exhibited significant improvements at week 24 in the KRG group. Other metabolic parameters were not altered after 24 weeks in both groups. In the subgroup analysis, CPT levels were improved in those with a longer diabetes duration or who already had neuropathy at the beginning of the study, and insulin resistance was improved in patients with a shorter diabetes duration. Conclusion. Twentyfour week administration of KRG in T2DM patients resulted in a significant improvement in neuropathy, especially in those with a longer diabetes duration. A further, larger population study with a longer follow-up period is warranted to verify the effects of KRG on diabetic neuropathy.
\end{abstract}

\section{Introduction}

Type 2 diabetes is the fastest-growing metabolic disease in the world, which involves various organs [1]. Chronic hyperglycemia leads to a damage in small blood vessels in peripheral nerves, kidney, and retina as well as in larger blood vessels of the heart and the brain. These chronic diabetic microvascular and macrovascular complications lead to significant mortality and morbidity in diabetes patients. Among them, diabetic neuropathy is the most common microvascular complication affecting as many as $50 \%$ of diabetes patients, and it is the leading cause of nontraumatic limb amputation [2-4].

Measurement of current perception threshold (CPT) using the Neurometer ${ }^{\circledR}$ has been proven to be a reliable method to assess diabetic neuropathy by quantifying sensory fiber function. The CPT test is widely used in clinical practice for being noninvasive and being able to detect not only advanced severe neuropathy but also early asymptomatic neuropathy compared to other sensory tests [5-7]. Despite many experimental and clinical studies, there is no effective treatment that directly affects the pathogenesis of diabetic neuropathy. We use $\alpha$-lipoic acid to reduce oxygen free radicals, and anticonvulsants such as gabapentin and pregabalin for pain relief in painful neuropathy, but their effectiveness and persistence are limited $[2,8,9]$.

Ginseng (Panax ginseng Meyer) roots have been widely used as an herbal treatment in East Asia for more than 2000 years. Korean Red Ginseng (KRG) is a popular traditional herb that is known to have beneficial effects in 
obesity, postmenopausal symptoms, cholesterol levels, and cardiovascular diseases. There are several animal and human studies to support these effects $[10,11]$. Many Type 2 diabetes patients also take KRG as a complementary and alternative medicine for better glycemic control and health promotion [12]. Various animal and clinical studies have been conducted, and KRG has been shown to improve postprandial glucose and insulin sensitivity in several studies $[13,14]$.

Such effects of KRG are thought to be due to antiinflammatory and antioxidant activities [15-17]. Chronic hyperglycemia with consequent oxidative stress, advanced glycation end product (AGE), and various inflammatory responses are known to mediate diabetic complications. Several animal studies have demonstrated that Red Ginseng can reduce diabetic microvascular complications and brain neuronal damage by reducing oxidative stress and advanced glycation end products (AGE) [18-20].

However, human studies on the effects of Red Ginseng on diabetic complications are lacking and the follow-up period is short. In this study, we investigated the effects of KRG administration on diabetic neuropathy in Type 2 diabetes patients.

\section{Research Design and Methods}

2.1. Subjects. A total of 70 Type 2 diabetes patients who visited the Gangnam Severance Hospital between April 2016 and July 2017 were enrolled in this single-center, doubleblind, randomized, placebo controlled trial. Eligible patients were men and women between the ages of 19 and 75 who were diagnosed with Type 2 diabetes more than 6 months prior and those taking oral antidiabetic agents at the time of enrollment with an unchanged dose or type of drug within the last 3 months. Patients with HbAlc $>10 \%$, eGFR $<30$ $\mathrm{ml} / \mathrm{min} / 1.73 \mathrm{~m}^{2}$, AST/ALT $>3$ times greater the upper normal limit, taking glucocorticoids or any herbal medicine within the past 3 months, chronic inflammatory disease in the active phase or acute infection status, and pregnant or lactating were excluded. Enrolled subjects were randomized using a computer-generated randomization table.

2.2. Design. Subjects were randomized to receive KRG extract tablets or placebos for 24 weeks. They were instructed to take 2 KRG extract tablets, with each tablet containing 500 milligrams of KRG extract powder, which is equivalent to taking $3 \mathrm{~g}$ of KRG extract per day. The components of KRG are as follows: $0.37 \mathrm{mg} / \mathrm{g}$ of ginsenoside $\mathrm{Rg} 1,0.49 \mathrm{mg} / \mathrm{g}$ of ginsenoside Re, $1.23 \mathrm{mg} / \mathrm{g}$ of ginsenoside Rf, $2.35 \mathrm{mg} / \mathrm{g}$ of ginsenoside $\mathrm{Rh} 1,3.06 \mathrm{mg} / \mathrm{g}$ of ginsenoside $\mathrm{Rg} 2 \mathrm{~s}, 4.43 \mathrm{mg} / \mathrm{g}$ of ginsenoside $\mathrm{Rb} 1,2.01 \mathrm{mg} / \mathrm{g}$ of ginsenoside Rc, $1.71 \mathrm{mg} / \mathrm{g}$ of ginsenoside $\mathrm{Rb} 2,0.86 \mathrm{mg} / \mathrm{g}$ of ginsenoside $\mathrm{Rd}, 3.27 \mathrm{mg} / \mathrm{g}$ of ginsenoside $\mathrm{Rg} 3 \mathrm{~s}, 1.02 \mathrm{mg} / \mathrm{g}$ of ginsenoside $\mathrm{Rg} 3 \mathrm{r}$, and $88.5 \mathrm{mg} / \mathrm{g}$ of acid polysaccharide. The HPLC picture of KRG components is shown in Figure 1. The placebo group took 2 tablets that were made from the same corn starch and cellulose as the KRG tablets in the same shape and size with the addition of Red Ginseng flavor twice a day. The KRG and placebo tablets were manufactured and supplied by the Korea Ginseng Corporation (Seoul, Korea). No other medication was prescribed, and the patients' original diabetes medication remained unchanged. All subjects, clinical investigators, and outcome assessors were blind to treatment allocation.

All participants provided informed consent, and the study was approved by the Institutional Review Board of Gangnam Severance Hospital. The study was registered on ISRCTN17039603.

\subsubsection{Anthropometric Parameters and Biochemical Profiles.} Body weight and height were measured in the morning with participants wearing light clothing. Body mass index (BMI) was calculated as body weight in kilograms divided by height in meters squared $\left(\mathrm{kg} / \mathrm{m}^{2}\right)$.

Blood samples were taken from the antecubital vein after at least 8 hours of fasting at weeks 0,12 , and 24 . The fasting plasma glucose concentrations were measured by a standard glucose oxidase method (747 Automatic Analyzer, Hitachi, Tokyo, Japan). Fasting serum insulin was determined by chemiluminescence (RIA Kit, Daiichi, Japan), and HbAlc was measured by high-performance liquid chromatography (VARIANT II, Bio-Rad, Hercules, CA, USA). A standard $75 \mathrm{~g}$ oral glucose tolerance test (OGTT) was also performed, and plasma glucose and insulin levels were measured in venous blood collected at 0, 60, and 120 minutes after ingestion. Total cholesterol, high-density lipoprotein (HDL) cholesterol, low-density lipoprotein (LDL) cholesterol, and triglycerides were measured enzymatically using a chemical analyzer (Daiichi, Hitachi 747, Japan). Apolipoprotein A-1 and apolipoprotein $\mathrm{B}$ were measured through an immunoturbidimetric method (Cobas Integra 800 Analyzer; Roche Diagnostics) using standard procedures.

Serum blood urea nitrogen (BUN) and creatinine $(\mathrm{Cr})$ levels were measured enzymatically using a chemical analyzer (AU5800, Beckman Coulter, Inc., Brea, CA, USA). Serum AST and ALT were measured for the liver function test (AU5800, Beckman Coulter, Inc., Brea, CA, USA).

2.2.2. Insulin Resistance. Insulin resistance was assessed by the HOMA equation as follows: Homeostasis Model Assessment of Insulin Resistance $(\mathrm{HOMA}-\mathrm{IR})=[$ fasting glucose $(\mathrm{mg} / \mathrm{dl})$ $\times$ fasting insulin $(\mathrm{mcIU} / \mathrm{ml}) / 405$.

2.2.3. Diabetic Neuropathy Assessment. Diabetic peripheral neuropathy was assessed using the current perception thresholds (CPT) test by Neurometer ${ }^{\circledR}$ (Neurotron, Inc., Baltimore, MD, USA). It was measured at the beginning of the study (baseline) and after 24 weeks of medication.

The patient was to sit in a comfortable position with two electrodes coated with conductive gel attached to unilateral dorsal surfaces of the distal phalanges of the index finger and great toe. The current was increased (to a maximum of $9.99 \mathrm{~mA}$ ) until the patient began to feel the current at the skin where the electrodes are attached. The current was then terminated, decreased by $0.08 \mathrm{~mA}$, and reapplied. The patient was tested with three different alternating frequencies of electrical stimulus $(2000,250$, and $5 \mathrm{~Hz})$. At each frequency, the 


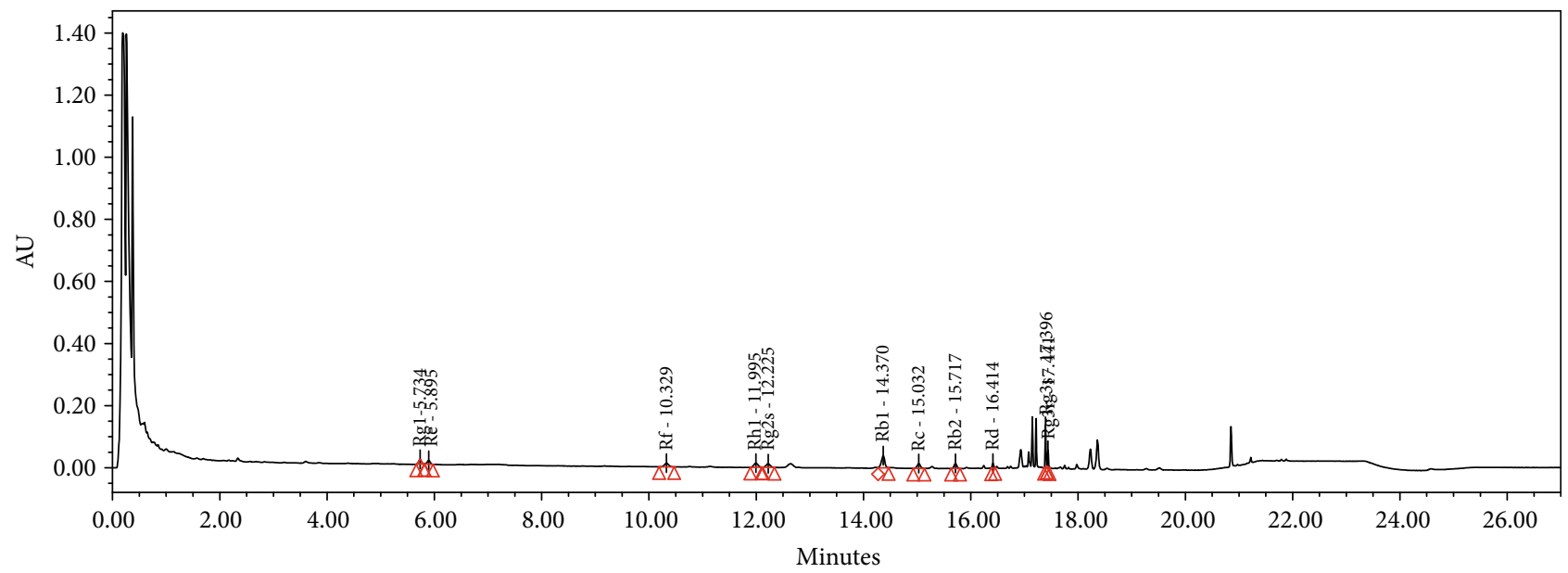

(a)

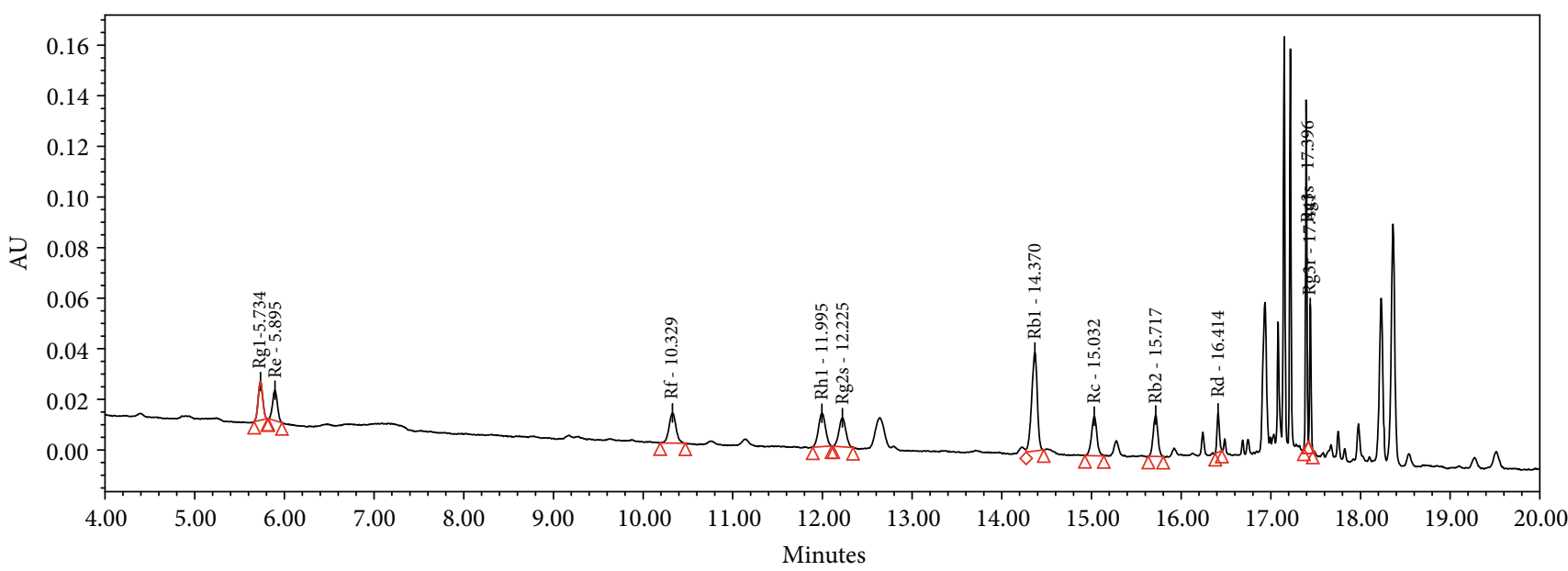

(b)

FIgURe 1: HPLC picture of KRG ginsenosides. An expanded version of (a) is shown in (b).

current was slowly increased until the subject first reported the perception of sensation. The current was then decreased and reincreased until a consistent threshold is measured [6].

2.2.4. Complication-Related Mediators. The serum levels of 8-epi-PGF2 $\alpha$ (Cloud-Clone Corp., CEA749Ge), highsensitivity CRP (CusaBio, CSB-E08617h), TNF- $\alpha$ (CloudClone Corp., SEA133Hu), and AGEs (Cloud-Clone Corp., CEB353Ge) were quantified by enzyme-linked immunosorbent assay. Blood samples were centrifuged immediately ( $1600 \mathrm{~g}, 10$ minutes), and the serum was stored at $-70^{\circ} \mathrm{C}$ until analysis. All procedures were performed according to the manufacturer's instructions.

2.3. Statistical Analysis. The primary outcomes were changes of CPT levels at week 24. Secondary outcomes were changes in fasting plasma glucose, $\mathrm{HbA1c}$, and various metabolic and inflammatory mediators of diabetic complications at weeks 0 and 24 .

Statistical analysis was performed using SPSS version 23.0 for Windows. All data were presented as mean \pm SD. The independent $t$-test was used to compare changes from baseline to 24 weeks between the two groups. The paired $t$-test was used to compare baseline data with changes at 24 weeks for each group. A value of $p<0.05$ was considered statistically significant.

\section{Results}

3.1. Patient Characteristics. During the study period, 9 patients dropped out due to a lack of time, lack of compliance, and adverse effects. A total of 61 patients (30 patients in the KRG group and 31 in the placebo group) completed the study (Figure 2). The patients' baseline characteristics are outlined in Table 1 . The participants' mean age was 59 years, mean BMI was $25 \mathrm{~kg} / \mathrm{m}^{2}$, and mean diabetes duration was 12 years. Approximately one-third of the patients were taking one type of oral hypoglycemic agent and two-thirds were taking more than two agents. There were no significant differences between the two groups in terms of smoking and alcohol consumption.

3.2. Changes in Metabolic Parameters. There were no differences in BMI and blood pressure after 24 weeks of 


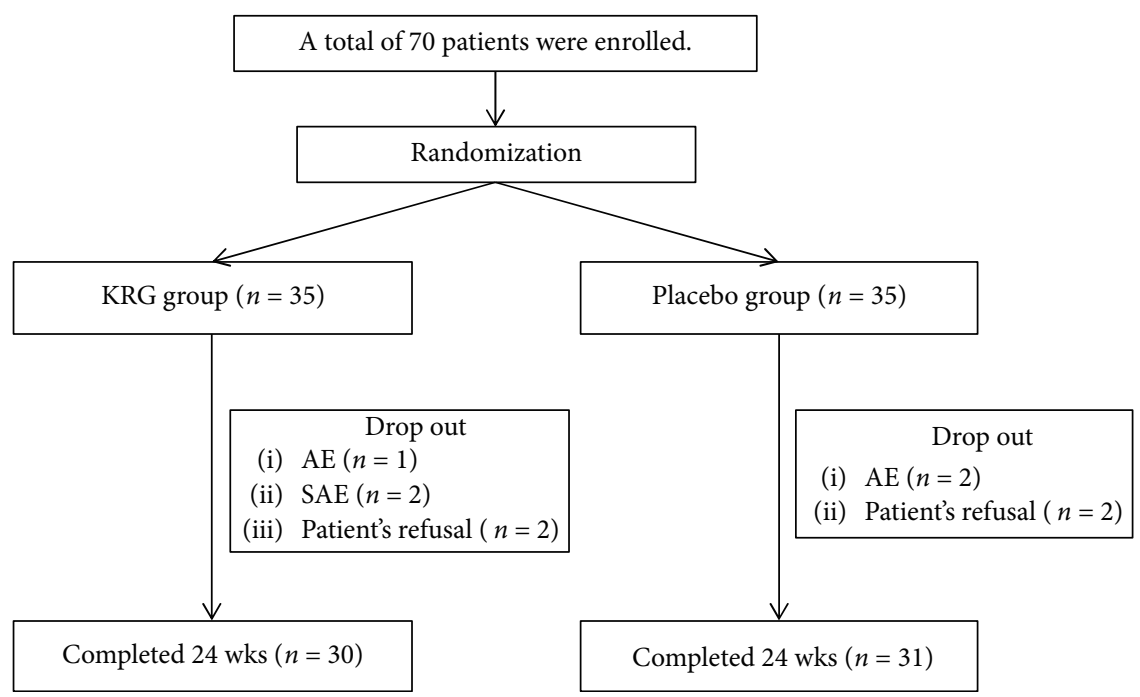

FIGURE 2: Trial flow.

TABLE 1: Subject characteristics at baseline.

\begin{tabular}{lccc}
\hline & KRG group $(n=30)$ & Placebo group $(n=31)$ & $p$ value $^{\mathrm{a}}$ \\
\hline Gender (M/F) & $17 / 13$ & $19 / 12$ & $\mathrm{NS}^{\mathrm{a}}$ \\
Age (years) & $59.3 \pm 8.79$ & $59.7 \pm 7.22$ & $\mathrm{NS}$ \\
BMI $\left(\mathrm{kg} / \mathrm{m}^{2}\right)$ & $24.7 \pm 2.93$ & $12.6 \pm 2.82$ & $\mathrm{NS}$ \\
DM duration (years) & $11.6 \pm 6.80$ & & \\
DM medication & & $11(35.5 \%)$ & $8(25.8 \%)$ \\
Monotherapy & $9(30.0 \%)$ & $12(38.7 \%)$ \\
Dual therapy & $13(43.3 \%)$ & $132.2 \pm 16.77$ \\
Triple therapy & $18(26.7 \%)$ & $80.8 \pm 12.03$ \\
SBP (mmHg) & $131.6 \pm 15.64$ & & $\mathrm{NS}$ \\
\hline DBP (mmHg) & $77.7 \pm 8.71$ & $20(64.5 \%)$ \\
Smoking & & $5(16.1 \%)$ \\
Nonsmoker & $16(53.3 \%)$ & $6(19.4 \%)$ \\
Ex-smoker & $3(10.0 \%)$ & & $\mathrm{NS}^{\mathrm{b}}$ \\
Current smoker & $11(36.7 \%)$ & $22(71.0 \%)$ \\
Alcohol & & $9(29.0 \%)$ \\
No & $22(73.3 \%)$ & $\mathrm{NS}^{\mathrm{b}}$ \\
Yes & $8(26.7 \%)$ & $\mathrm{NS}^{\mathrm{b}}$ \\
\hline
\end{tabular}

Data are presented as mean $\pm \mathrm{SD} .{ }^{a}$ Analyzed by independent $t$-tests and the $p$ value represents the comparison to the placebo group. ${ }^{\mathrm{b}}$ Analyzed by Chi-square. KRG: Korean Red Ginseng; BMI: body mass index; SBP: systolic blood pressure; DBP: diastolic blood pressure.

medication. Also, there were no significant differences regarding $\mathrm{HbAlc}$, fasting plasma glucose, and insulin levels in both groups. As an index of insulin resistance, HOMAIR was elevated after 24 weeks in both the KRG and placebo groups, but was not statistically significant. Lipid profiles (total cholesterol, triglyceride, apolipoprotein A1, apolipoprotein B, HDL, and LDL-cholesterol) also exhibited no statistically significant changes after 24 weeks of drug administration (Table 2).

3.3. Changes in CPT and Diabetic Complication-Related Mediators. CPT was significantly improved in both the left and right lower extremities at various frequencies in the
KRG group, while most of the CPT did not show statistically significant improvement in the placebo group (Table 3).

8 -epi-PGF $2 \alpha$, advanced glycation end product (AGE), and inflammatory markers (hsCRP, IL-6, and TNF- $\alpha$ ) were not significantly altered in both the KRG and placebo groups (Table 3).

3.4. Subgroup Analysis. Subjects were divided into subgroups according to the duration of diabetes and the presence of diabetic microvascular complications.

In subjects with diabetes duration of less than 5 years, fasting insulin decreased from $11.1 \pm 4.1$ to $6.3 \pm 2.1 \mathrm{mcIU} / \mathrm{ml}$, and HOMA-IR improved significantly from $3.39 \pm 1.2$ to 
TABLE 2: Anthropometric and biochemical profile.

\begin{tabular}{|c|c|c|c|c|c|c|}
\hline & \multicolumn{3}{|c|}{ KRG group $(n=30)$} & \multicolumn{3}{|c|}{ Placebo group $(n=31)$} \\
\hline & Week 0 & Week 24 & Within-group $p$ value & Week 0 & Week 24 & Within-group $p$ value \\
\hline BMI $\left(\mathrm{kg} / \mathrm{m}^{2}\right)$ & $24.8 \pm 2.96$ & $24.5 \pm 2.75$ & NS & $24.4 \pm 2.68$ & $24.2 \pm 2.76$ & NS \\
\hline $\mathrm{SBP}(\mathrm{mmHg})$ & $132 \pm 15.91$ & $133 \pm 15.21$ & NS & $132 \pm 16.88$ & $130 \pm 19.18$ & NS \\
\hline $\mathrm{DBP}(\mathrm{mmHg})$ & $78 \pm 8.77$ & $80 \pm 9.13$ & NS & $80 \pm 12.11$ & $79 \pm 13.84$ & NS \\
\hline HbAlc (\%) & $7.4 \pm 0.79$ & $7.5 \pm 0.83$ & NS & $7.4 \pm 0.70$ & $7.7 \pm 0.85$ & NS \\
\hline $\mathrm{FPG}(\mathrm{mg} / \mathrm{dl})$ & $143 \pm 30.41$ & $150 \pm 31.98$ & NS & $153 \pm 30.09$ & $159 \pm 39.33$ & NS \\
\hline $2 \mathrm{~h} \mathrm{Glc}(\mathrm{mg} / \mathrm{dl})$ & $305 \pm 65.31$ & $307 \pm 74.60$ & NS & $301 \pm 67.49$ & $306 \pm 79.25$ & NS \\
\hline Ins (mcIU/ml) & $7.6 \pm 4.51$ & $7.3 \pm 4.34$ & NS & $7.8 \pm 5.08$ & $7.8 \pm 5.12$ & NS \\
\hline $2 \mathrm{~h}$ Ins $(\mathrm{mcIU} / \mathrm{ml})$ & $31.6 \pm 17.93$ & $31.6 \pm 20.03$ & NS & $32.1 \pm 23.53$ & $29.4 \pm 21.12$ & NS \\
\hline HOMA-IR & $2.6 \pm 1.71$ & $2.7 \pm 1.84$ & NS & $2.9 \pm 1.80$ & $3.1 \pm 2.42$ & NS \\
\hline $\mathrm{TC}(\mathrm{mg} / \mathrm{dl})$ & $171 \pm 36.14$ & $179 \pm 41.51$ & NS & $170 \pm 33.64$ & $180 \pm 39.62$ & NS \\
\hline $\mathrm{TG}(\mathrm{mg} / \mathrm{dl})$ & $167 \pm 130.82$ & $160 \pm 93.52$ & NS & $141 \pm 60.90$ & $182 \pm 177.19$ & NS \\
\hline HDL-C (mg/dl) & $48 \pm 10.44$ & $49 \pm 8.65$ & NS & $50 \pm 9.56$ & $50 \pm 11.16$ & NS \\
\hline LDL-C (mg/dl) & $114 \pm 28.80$ & $119 \pm 34.27$ & NS & $113 \pm 29.35$ & $117 \pm 30.75$ & NS \\
\hline Apo A1 (mg/dl) & $139.3 \pm 17.88$ & $140.0 \pm 17.66$ & NS & $146.8 \pm 20.34$ & $145.3 \pm 22.87$ & NS \\
\hline Apo B (mg/dl) & $89.6 \pm 19.29$ & $95.6 \pm 25.84$ & NS & $90.4 \pm 21.49$ & $95.2 \pm 22.53$ & NS \\
\hline
\end{tabular}

BMI: body mass index; SBP: systemic blood pressure; DBP: diastolic blood pressure; FPG: fasting plasma glucose; $2 \mathrm{~h}$ Glc: 2 -hour glucose; Ins: fasting insulin; $2 \mathrm{~h}$ Ins: 2-hour insulin; TC: total cholesterol; TG: triglyceride; HDL-C: HDL-cholesterol; LDL-C: LDL-cholesterol; Apo A1: apolipoprotein A1; Apo B: apolipoprotein B.

TABLE 3: Diabetic microvascular complication studies.

\begin{tabular}{|c|c|c|c|c|c|c|}
\hline & \multicolumn{3}{|c|}{ KRG group $(n=30)$} & \multicolumn{3}{|c|}{ Placebo group $(n=31)$} \\
\hline & Week 0 & Week 24 & Within-group $p$ value & Week 0 & Week 24 & Within-group $p$ value \\
\hline \multicolumn{7}{|l|}{ CPT of Lex, right } \\
\hline $2000 \mathrm{~Hz}$ & $306.07 \pm 82$ & $266.79 \pm 81$ & $0.010^{*}$ & $295.54 \pm 87$ & $256.79 \pm 80$ & NS \\
\hline $250 \mathrm{~Hz}$ & $135.64 \pm 40$ & $110.07 \pm 36$ & $0.007^{*}$ & $140.68 \pm 55$ & $118.93 \pm 35$ & NS \\
\hline $5 \mathrm{~Hz}$ & $88.61 \pm 38$ & $76.07 \pm 25$ & NS & $93.11 \pm 33$ & $82.79 \pm 33$ & NS \\
\hline \multicolumn{7}{|l|}{ CPT of Lex, left } \\
\hline $2000 \mathrm{~Hz}$ & $300.79 \pm 82$ & $267.14 \pm 82$ & $0.023^{*}$ & $288.86 \pm 93$ & $266.86 \pm 72$ & NS \\
\hline $250 \mathrm{~Hz}$ & $130.07 \pm 40$ & $105.18 \pm 35$ & $0.005^{*}$ & $137.29 \pm 67$ & $107.39 \pm 35$ & $0.022^{*}$ \\
\hline $5 \mathrm{~Hz}$ & $85.54 \pm 25$ & $69.04 \pm 23$ & $0.007^{*}$ & $91.71 \pm 35$ & $70.96 \pm 27$ & $0.004^{*}$ \\
\hline PGF $2 \alpha(\mathrm{pg} / \mathrm{ml})$ & $132.48 \pm 109.50$ & $125.89 \pm 110.00$ & NS & $151.34 \pm 143.06$ & $137.73 \pm 122.23$ & NS \\
\hline hsCRP (mcg/ml) & $2.55 \pm 7.47$ & $0.61 \pm 0.26$ & NS & $1.56 \pm 2.51$ & $1.04 \pm 1.73$ & NS \\
\hline IL-6 (pg/ml) & $2.90 \pm 6.21$ & $2.09 \pm 1.75$ & NS & $1.86 \pm 1.48$ & $1.83 \pm 1.57$ & NS \\
\hline TNF- $\alpha(\mathrm{pg} / \mathrm{ml})$ & $10.70 \pm 20.82$ & $14.73 \pm 34.26$ & NS & $3.11 \pm 4.41$ & $2.96 \pm 4.33$ & NS \\
\hline $\mathrm{AGE}(\mathrm{mcg} / \mathrm{ml})$ & $2.01 \pm 1.06$ & $2.11 \pm 1.03$ & NS & $1.89 \pm 0.96$ & $1.93 \pm 0.94$ & NS \\
\hline
\end{tabular}

${ }^{*} p \leq 0.05$ is significant. CPT: current perception threshold; Lex: lower extremities; PGF2 $\alpha$ : isoprostane 8-epi PGF2 $\alpha$; hsCRP: high-sensitivity C-reactive protein; IL-6: interleukin 6; TNF- $\alpha$ : TNF-alpha; AGE: advanced glycation end product.

$2.08 \pm 0.8$ after KRG treatment $(p=0.044$ and $p=0.046$ respectively). In the patients with diabetes duration of more than 5 years, the CPT of lower extremities were significantly improved after KRG administration from $299.42 \pm 81$ to $258.92 \pm 86$ at $2000 \mathrm{~Hz}(p=0.012)$ and from $137.13 \pm 43$ to $106.96 \pm 36$ at $250 \mathrm{~Hz}(p=0.002)$, while there were no significant changes in the placebo group.

In patients who already had abnormal CPT at baseline, the CPT levels at $2000 \mathrm{~Hz}$ decreased significantly from
$323.24 \pm 91$ to $278.94 \pm 93(p=0.049)$, and the CPT levels at $250 \mathrm{~Hz}$ decreased significantly from $145.65 \pm 46$ to $119.35 \pm$ $36(p=0.028)$ after KRG administration. These changes were not observed in the placebo group.

3.5. Safety. There were no serious adverse effects in both the KRG and placebo groups. Three patients in the KRG group dropped out due to fever and gastrointestinal disturbance, foot pain, and deterioration of blood glucose. In the placebo 
group, two patients dropped out due to fever, palpitations, and fatigue. Serum AST and ALT levels did not deteriorate, and hypoglycemia did not occur during the study period in both groups.

\section{Discussion}

In this study, we investigated the effects of KRG on diabetic neuropathy in Type 2 diabetes patients. KRG is a Korean traditional herb that is becoming popular worldwide for its beneficial effects in obesity, insulin resistance, dyslipidemia, and cardiovascular diseases due to its antioxidant and antiinflammatory effects. Many diabetes patients take KRG for the above reasons. However, there has been no study that investigated its effect on diabetic neuropathy, which is the most common diabetic microvascular complication that does not have a definite pathogenic treatment option.

Twenty-four weeks of KRG administration resulted in improved peripheral neuropathy particularly in those with a longer diabetes duration and underlying neuropathy. Meanwhile, insulin sensitivity improved in those with a relatively short diabetes duration. We believe our study results have significance for its possible beneficial effects in diabetic neuropathy without serious adverse events.

Diabetic neuropathy is the most common diabetic chronic complication that results in pain, disability, and amputation. Its pathogenesis is not fully understood yet, but toxic effects of chronic hyperglycemia involving oxidative stress, polyol pathway, and advanced glycation end products (AGE) are known to play important roles [21-25]. Ginseng has been shown to have antioxidant and anti-inflammatory properties, and thus studies have been conducted to assess the effects of ginseng on diabetic microvascular complications including neuropathy, retinopathy, and nephropathy in diabetic animal models $[26,27]$. However, there has been no human study so far.

In this study, the CPT test, which is a quick, convenient, but also a reliable way to assess diabetic neuropathy was used [28]. The KRG group showed consistent and statistically significant improvements of CPT levels throughout different frequencies of stimulus. Meanwhile, the placebo group also showed improved CPT, but mostly it did not reach statistical significance. In particular, CPT levels at higher frequencies were significantly improved in the KRG group. The CPT levels at 2000 or $250 \mathrm{~Hz}$ are more clinically important than those at $5 \mathrm{~Hz}$, because it has been shown that the Neurometer ${ }^{\circledR}$ displays poor reproducibility at low frequencies like $5 \mathrm{~Hz}$ compared to 250 and $2000 \mathrm{~Hz}$ [29]. Also, it was notable that CPT was significantly improved in patients with longer diabetes duration and in those who already had an impaired CPT at baseline.

We have assessed various factors known to mediate chronic diabetic complications to investigate possible mechanisms for such improvement. We investigated AGE and various oxidative and inflammatory markers such as highsensitivity C-reactive protein, interleukin 6 , TNF- $\alpha$, and 8epi-PGF $2 \alpha$. However, they were not altered after 24 weeks of KRG and did not show significant correlations with changes in CPT. There have been many studies assessing the effects of natural compounds with antioxidant or anti-inflammatory effects on diabetic complications, and many showed benefits. For example, an animal study conducted on streptozotocin-induced diabetes mice demonstrated a decreased AGE level in urine and kidney as well as plasma TNF- $\alpha$ and reduced renal damage after KRG administration [16].

However, like our study, most of the human studies conducted with natural compounds on diabetic neuropathy failed to elucidate the underlying mechanism [26]. First of all, compared to diabetic nephropathy, it is harder to assess diabetic neuropathy and its mechanism is more complex. Also, in contrast to pharmacologically manufactured products with high potency and predictable pharmacodynamics, natural compounds like KRG tend to have diverse pleiotropic effects rather than one strong specific effect. Therefore, although their properties can be observed in in vivo experiments, it may be too moderate to be seen at the human blood level. In other words, the effect of KRG on neuropathy may have resulted not from one strong mechanism but rather from multiple mechanisms that interact with each other.

There have been inconsistent data for the effects of KRG on blood glucose control, insulin resistance, and various metabolic parameters. In this study, the administration of KRG for 24 weeks did not affect patient BMI, blood pressure, and blood glucose. Regarding insulin resistance, changes in fasting insulin and HOMA-IR were not significantly different between the two groups. However, in the subgroup analysis with patients whose duration of diabetes mellitus was relatively short (less than 5 years), fasting insulin significantly decreased from 11.1 to $6.3 \mathrm{mcIU} / \mathrm{ml}$ and HOMA-IR significantly improved from 3.39 to $2.08(p=0.044$ and $p=$ 0.046 , respectively). These results are in line with previous studies that KRG improves peripheral insulin resistance $[10,14,30,31]$.

In terms of safety, there was no hepatotoxicity, nephrotoxicity, or hypoglycemia in the KRG group. Among the 3 patients in the KRG group who dropped out due to adverse effects, one was admitted to the hospital due to deterioration in blood glucose level. However, this was because the patient did not properly take the prescribed antidiabetic drugs and had an uncontrolled diet. Another patient in the KRG group complained of fever and gastrointestinal disturbance, but there was one patient with similar symptoms in the placebo group. The third patient complained of foot pain, but there was no sign of diabetic foot. In the placebo group, one patient dropped out due to fever and fatigue, while another patient exhibited elevated liver enzymes.

There are several limitations to this study. First of all, the number of study subjects was small, and the study duration was relatively short to assess chronic diabetic complications. However, this was the first randomized controlled trial to assess the effects of KRG on diabetic neuropathy in Type 2 diabetes patients. Secondly, the underlying mechanism for improved CPT and insulin resistance following KRG administration had not been elucidated. Thirdly, there is no data after the discontinuation of KRG to examine the reversal effect. Also, all the subjects took the same dose of KRG, which is the commercially available dose in Korea, regardless of 
their body weight. This study would have observed more precise KRG effects if the dose was titrated to body weight. Lastly, subjects with renal or hepatic dysfunction were excluded from the study, and therefore, the effects and safety of KRG cannot be generalized.

In conclusion, in this first randomized controlled trial to assess the effects of KRG on chronic diabetic neuropathy, a 24-week administration of KRG significantly improved markers of diabetic neuropathy especially in those who already had neuropathy or a long diabetes duration. In addition, it also improved insulin resistance in patients with a short diabetes duration. Considering that diabetic microvascular complication is a chronic progressive disease, a further, larger population study with a longer follow-up period is warranted to verify and understand the effects of KRG on diabetic complications.

\section{Abbreviations}

AGE: Advanced glycation end products

CPT: Current perception threshold

KRG: Korean Red Ginseng.

\section{Data Availability}

The data used to support the findings of this study are available from the corresponding author upon request.

\section{Disclosure}

The abstract was presented at the 48th EASD Annual Meeting of the European Association for the Study of Diabetes.

\section{Conflicts of Interest}

The authors declare no conflicts of interest.

\section{Authors' Contributions}

K.P. researched data and wrote the manuscript. J.K. contributed to collecting data. C.W.A., S.K., J.S.P., and Y.K. contributed to interpreting the findings and editing the manuscript. J.S.N. is the guarantor of this work and, as such, had full access to all the data in the study and takes responsibility for the integrity of the data and the accuracy of the data analysis.

\section{Acknowledgments}

This study was financially supported by the Korea Ginseng Corporation.

\section{References}

[1] S. Wild, G. Roglic, A. Green, R. Sicree, and H. King, "Global prevalence of diabetes: estimates for the year 2000 and projections for 2030," Diabetes Care, vol. 27, no. 5, pp. 1047-1053, 2004.
[2] A. J. Boulton, A. I. Vinik, J. C. Arezzo et al., "Diabetic neuropathies: a statement by the American Diabetes Association," Diabetes Care, vol. 28, no. 4, pp. 956-962, 2005.

[3] J. C. Won, H. S. Kwon, C. H. Kim et al., "Prevalence and clinical characteristics of diabetic peripheral neuropathy in hospital patients with type 2 diabetes in Korea," Diabetic Medicine, vol. 29, no. 9, pp. e290-e296, 2012.

[4] M. Yorek, R. A. Malik, N. A. Calcutt, A. Vinik, and S. Yagihashi, "Diabetic neuropathy: new insights to early diagnosis and treatments," Journal Diabetes Research, vol. 2018, article 5378439, 3 pages, 2018.

[5] J. H. Park and J. C. Won, "Patterns of nerve conduction abnormalities in patients with type 2 diabetes mellitus according to the clinical phenotype determined by the current perception threshold," Diabetes and Metabolism Journal, vol. 42, no. 6, pp. 519-528, 2018.

[6] G. V. Inceu and I. A. Veresiu, "Measurement of current perception thresholds using the Neurometer ${ }^{\circledR}$ - applicability in diabetic neuropathy," Medicine and Pharmacy Reports, vol. 88, no. 4, pp. 449-452, 2015.

[7] R. Matsutomo, K. Takebayashi, and Y. Aso, "Assessment of peripheral neuropathy using measurement of the current perception threshold with the neurometer in patients with type 2 diabetes mellitus," The Journal of International Medical Research, vol. 33, no. 4, pp. 442-453, 2005.

[8] H. Garcia-Alcala, C. I. Santos Vichido, S. Islas Macedo et al., "Treatment with $\alpha$-Lipoic Acid over 16 Weeks in Type 2 Diabetic Patients with Symptomatic Polyneuropathy Who Responded to Initial 4-Week High-Dose Loading," Journal Diabetes Research, vol. 2015, article 189857, 8 pages, 2015.

[9] A. Mimenza Alvarado and S. Aguilar Navarro, "Clinical trial assessing the efficacy of gabapentin plus B complex (B1/B12) versus pregabalin for treating painful diabetic neuropathy," Journal Diabetes Research, vol. 2016, article 4078695, 8 pages, 2016.

[10] H. Lee, J. Choi, S. S. Shin, and M. Yoon, "Effects of Korean red ginseng (Panax ginseng) on obesity and adipose inflammation in ovariectomized mice," Journal of Ethnopharmacology, vol. 178, pp. 229-237, 2016.

[11] S. Y. Kim, S. K. Seo, Y. M. Choi et al., "Effects of red ginseng supplementation on menopausal symptoms and cardiovascular risk factors in postmenopausal women: a double-blind randomized controlled trial," Menopause, vol. 19, no. 4, pp. 461466, 2012.

[12] M. S. Lee, M. S. Lee, H. J. Lim, and S. R. Moon, "Survey of the use of complementary and alternative medicine among Korean diabetes mellitus patients," Pharmacoepidemiology and Drug Safety, vol. 13, no. 3, pp. 167-171, 2004.

[13] M. R. Oh, S. H. Park, S. Y. Kim et al., "Postprandial glucose-lowering effects of fermented red ginseng in subjects with impaired fasting glucose or type 2 diabetes: a randomized, double-blind, placebo-controlled clinical trial," $B M C$ Complementary and Alternative Medicine, vol. 14, no. 1, p. 237, 2014.

[14] V. Vuksan, M. K. Sung, J. L. Sievenpiper et al., "Korean red ginseng (Panax ginseng) improves glucose and insulin regulation in well-controlled, type 2 diabetes: Results of a randomized, double-blind, placebo-controlled study of efficacy and safety," Nutrition, Metabolism, and Cardiovascular Diseases, vol. 18, no. 1, pp. 46-56, 2008.

[15] J. Y. Kim, J. Y. Park, H. J. Kang, O. Y. Kim, and J. H. Lee, "Beneficial effects of Korean red ginseng on lymphocyte DNA 
damage, antioxidant enzyme activity, and LDL oxidation in healthy participants: a randomized, double-blind, placebocontrolled trial," Nutrition Journal, vol. 11, no. 1, p. 47, 2012.

[16] S. K. Seo, Y. Hong, B. H. Yun et al., "Antioxidative effects of Korean red ginseng in postmenopausal women: a doubleblind randomized controlled trial," Journal of Ethnopharmacology, vol. 154, no. 3, pp. 753-757, 2014.

[17] Y. Y. Lee, E. Saba, M. Irfan et al., "The anti-inflammatory and anti-nociceptive effects of Korean black ginseng," Phytomedicine, vol. 54, pp. 169-181, 2019.

[18] J. Y. Ban, S. W. Kang, J. S. Lee, J. H. Chung, Y. G. Ko, and H. S. Choi, "Korean red ginseng protects against neuronal damage induced by transient focal ischemia in rats," Experimental and Therapeutic Medicine, vol. 3, no. 4, pp. 693698, 2012.

[19] H. Y. Quan, D. Y. Kim, and S. H. Chung, "Korean red ginseng extract alleviates advanced glycation end product-mediated renal injury," Journal of Ginseng Research, vol. 37, no. 2, pp. 187-193, 2013.

[20] S. Sen, S. Chen, Y. Wu, B. Feng, E. K. Lui, and S. Chakrabarti, "Preventive effects of North American ginseng (Panax quinquefolius) on diabetic retinopathy and cardiomyopathy," Phytotherapy Research, vol. 27, no. 2, pp. 290-298, 2013.

[21] C. Ferri, G. Croce, V. Cofini et al., "C-reactive protein: interaction with the vascular endothelium and possible role in human atherosclerosis," Current Pharmaceutical Design, vol. 13, no. 16, pp. 1631-1645, 2007.

[22] Y. Mugabo, L. Li, and G. Renier, "The connection between Creactive protein (CRP) and diabetic vasculopathy. Focus on preclinical findings," Current Diabetes Reviews, vol. 6, no. 1, pp. 27-34, 2010.

[23] D. M. Niedowicz and D. L. Daleke, "The role of oxidative stress in diabetic complications," Cell Biochemistry and Biophysics, vol. 43, no. 2, pp. 289-330, 2005.

[24] V. P. Singh, A. Bali, N. Singh, and A. S. Jaggi, "Advanced glycation end products and diabetic complications," The Korean Journal of Physiology \& Pharmacology, vol. 18, no. 1, pp. 114, 2014.

[25] A. K. Schreiber, C. F. Nones, R. C. Reis, J. G. Chichorro, and J. M. Cunha, "Diabetic neuropathic pain: physiopathology and treatment," World Journal of Diabetes, vol. 6, no. 3, pp. 432444, 2015.

[26] M. Galuppo, S. Giacoppo, P. Bramanti, and E. Mazzon, "Use of natural compounds in the management of diabetic peripheral neuropathy," Molecules, vol. 19, no. 3, pp. 2877-2895, 2014.

[27] L. He, H. Wang, C. Gu, X. He, L. Zhao, and X. Tong, "Administration of traditional Chinese blood circulation activating drugs for microvascular complications in patients with type 2 diabetes mellitus," Journal Diabetes Research, vol. 2016, article 1081657, 9 pages, 2016.

[28] K. S. Park, Y. C. Kwon, M. Youn, Y.-S. Park, Y.-H. Hong, and J.-J. Sung, "Current perception threshold in diabetic sensory polyneuropathy with normal routine nerve conduction study," Annals of Clinical Neurophysiology, vol. 19, no. 2, pp. 125-130, 2017.

[29] B. C. Tsui, T. J. Shakespeare, D. H. Leung, J. H. Tsui, and G. N. Corry, "Reproducibility of current perception threshold with the Neurometer ${ }^{\circledR}$ vs the Stimpod NMS450 peripheral nerve stimulator in healthy volunteers: an observational study," Canadian Journal of Anaesthesia, vol. 60, no. 8, pp. 753-760, 2013.
[30] J. M. Cheon, D. I. Kim, and K. S. Kim, "Insulin sensitivity improvement of fermented Korean Red Ginseng (Panax ginseng) mediated by insulin resistance hallmarks in old-aged ob/ob mice," Journal of Ginseng Research, vol. 39, no. 4, pp. 331-337, 2015.

[31] S. H. Lee, H. J. Lee, Y. H. Lee et al., "Korean red ginseng (Panax ginseng) improves insulin sensitivity in high fat fed SpragueDawley rats," Phytotherapy Research, vol. 26, no. 1, pp. 142147, 2012. 


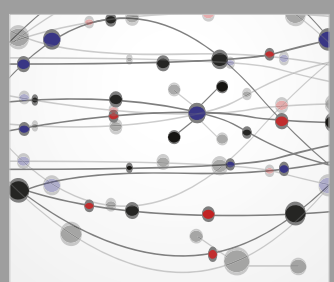

The Scientific World Journal
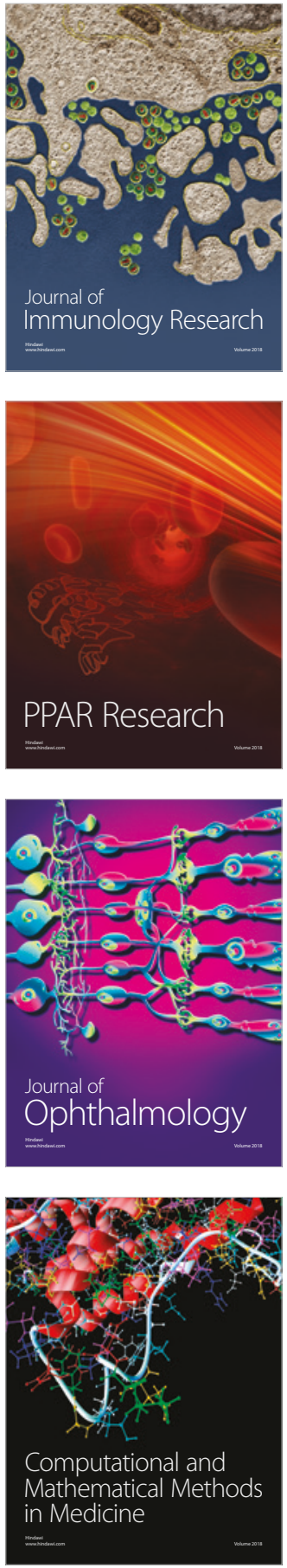

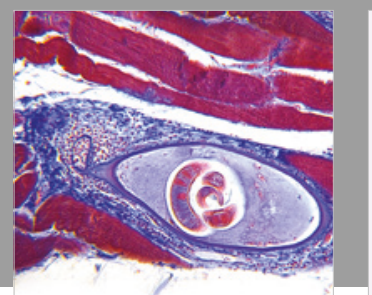

Gastroenterology Research and Practice

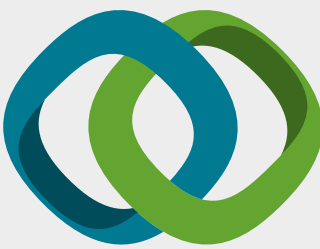

\section{Hindawi}

Submit your manuscripts at

www.hindawi.com
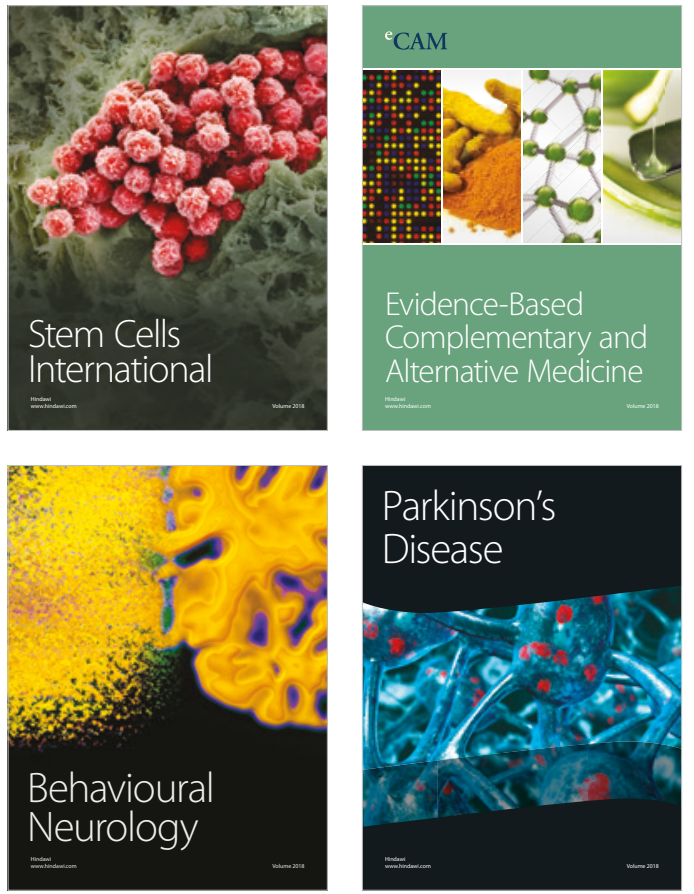

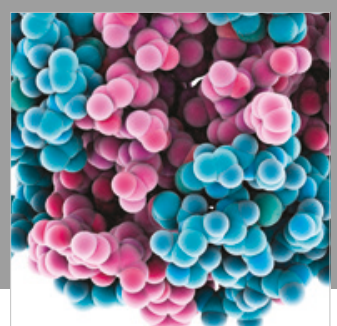

ournal of

Diabetes Research

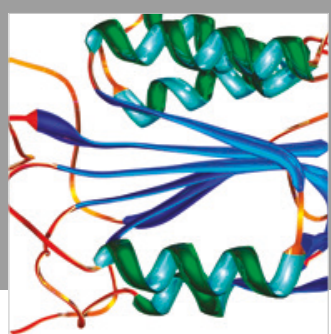

Disease Markers
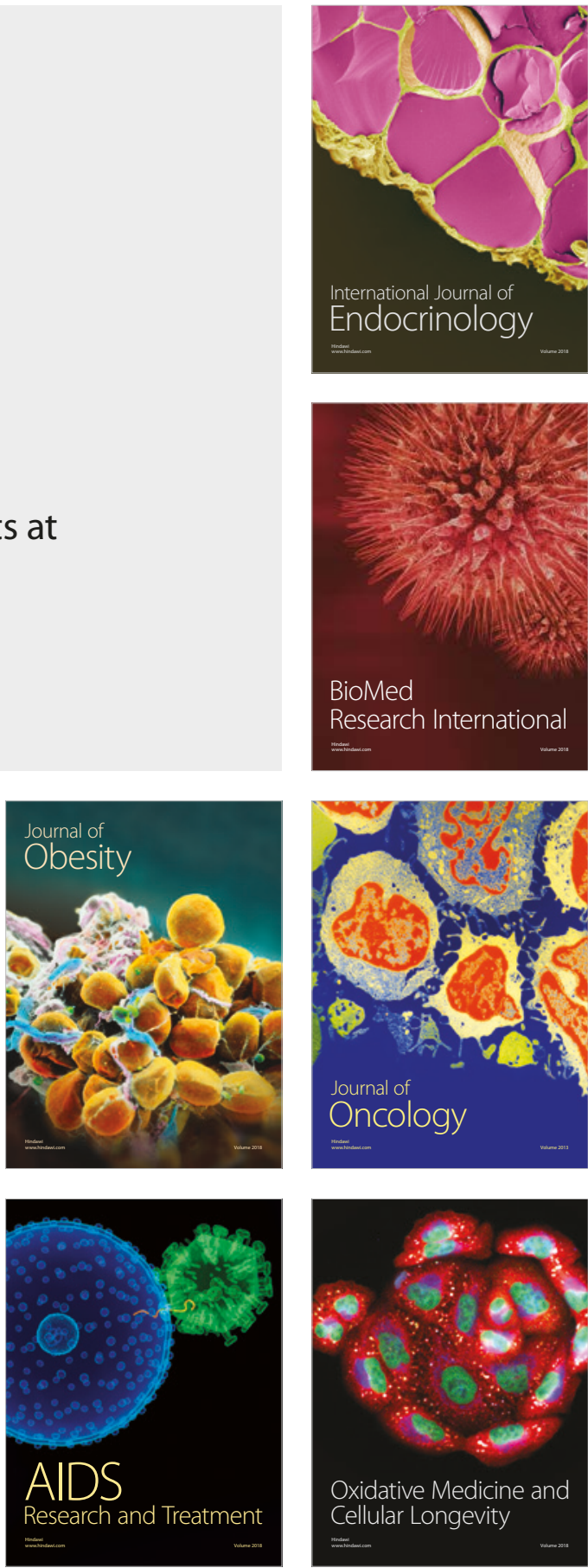